\title{
"Smart Microscopy": Feature Based Adaptive Sampling for Focused Ion Beam Scanning Electron Microscopy
}

Tim Dahmen ${ }^{1}$, Niels de Jonge ${ }^{2}$, Patrick Trampert ${ }^{1,3}$, Michael Engstler ${ }^{3}$, Christoph Pauly ${ }^{3}$, Frank Mücklich $^{3}$ and Philipp Slusallek ${ }^{1,3}$

1. German Research Center for Artificial Intelligence, Saarbrücken, Germany

2. INM - Leibniz Institute for New Materials, Saarbrücken, Germany

3. Saarland University, Saarbrücken, Germany

A new method for the image acquisition in scanning electron microscopy (SEM) was introduced. The method used adaptively increased pixel-dwell times to improve the signal-to-noise ratio (SNR) in areas of high detail. In areas of low detail, the electron dose was reduced on a per pixel basis, and a-posteriori image processing techniques were applied to remove the resulting noise. The technique was realized by scanning the sample twice. The first, quick scan used small pixel-dwell times to generate a first, noisy image using a low electron dose. This image was analyzed automatically, and a software algorithm generated a sparse pattern of regions of the image that require additional sampling. A second scan generated a sparse image of only these regions, but using a highly increased electron dose. By applying a selective low-pass filter and combining both datasets, a single image was generated. The resulting image exhibited a factor of $\approx 3$ better SNR than an image acquired with uniform sampling on a Cartesian grid and the same total acquisition time. This result implies that the required electron dose (or acquisition time) for the adaptive scanning method is a factor of ten lower than for uniform scanning.

The method presented in this study [1,2] started with the acquisition of an image at a low pixel-dwell time of $0.3 \mu \mathrm{s}$. It was analyzed automatically and classified in two regions. The first region ("filter region") showed smooth gradients, i.e. only low frequency variation of the gray values. The second region, the high interest area (HIA), consisted of strong discontinuities of the gray values, i.e. it contained the pixels showing edges and other sharp features. The classification was done in such a way that a fixed amount of pixels was classified as belonging to the HIA, typical values are $10 \%$ of all pixels, depending on the sample. The shape of the sample area was transferred to the microscope in the form of a stream file, and a second scan was performed for only the HIA, but with a highly increased pixel dwell time of $27 \mu \mathrm{s}$. Aggregated, the sample was scanned with an average dwell time of $3 \mu \mathrm{s}$ per pixel (0.3 $\mu$ s initial $+0.1 \times 27 \mu$ s sparse). Initial and sparse dataset were combined using a special filtering and composition method to form the final image.

In order to benchmark the method, a conventional scan of the same sample was performed using uniform scanning with the same pixel-dwell time of $3 \mu \mathrm{s}$ (Figure 1b). As image processing operations might also be applied to images recorded using uniform scanning, a anisotropic diffusion operator [3] was applied as an example of a state-of-the-art edge preserving smoothing filter (Figure 1c). As can be seen by comparing Figure 1a to Figure 1b, the image recorded using the adaptive sampling technique looks smoother and contains less image noise. An interesting benchmark is to compare the image acquired using adaptive sampling (Figure 1a) to the result obtained by combining uniform sampling at the same average pixel-dwell time and applying an anisotropic diffusion filter as an example of a stateof-the-art a-posteriori image processing methods (Figure 1c). As can be seen, the anisotropic diffusion filter is effective at removing noise, but also creates a slightly blurred image that misses even more details compared to the adaptive sampling scheme. 
Advantages of the method are two-fold. For the acquisition of a high resolution 3D dataset with FIB/SEM, the total acquisition time is one of the main limiting factors of the technique. Particularly for the acquisition of high-resolution 3D datasets, pixel-dwell times can dominate the total beam time of days or weeks and, thus, limit the feasibility of data acquisition. The proposed method allows reducing acquisition times by a factor of ten depending on the sample. Saving beam time is not the only motivation for reducing pixel-dwell times. Many samples, like biological samples, polymers, and ceramics, are sensitive to electron dose. For these cases, imaging means finding a compromise between beam-induced radiation damage and noise. The method proposed in this paper can reduce the average pixel-dwell time and, thus, helps to avoid radiation damage without introducing noise. However, the total electron dose will no longer be distributed evenly over the entire specimen, but focused at phase boundaries. While the effects of radiation damage are known for a range of different specimens, an open question is what happens in case of inhomogeneous spatial distribution of the radiation. The usefulness of the adaptive scanning scheme for the imaging of dose sensitive specimen will largely depend on the question how different kinds of specimens behave under locally focused radiation. [4]

\section{References:}

[1] Tim Dahmen et. al. Feature Adaptive Sampling for Scanning Electron Microscopy, under review Nature: Scientific Reports

[2] Dahmen, T. \& de Jonge, N. Verfahren und Vorrichtung zur Untersuchung von Proben durch ein Elektronen- oder Ionenstrahlmikroskop. German Patent No 102015114843.9 (2015).

[3] Weickert, J. Anisotropic diffusion in image processing. Image Rochester NY 256, 170 (1998).

[4] The authors acknowledge funding from European Research Project NOTOX (FP7-267038), the DFG grant IMCL (AOBJ: 600875) and the "Landesforschungsförderungsprogramm des Saarlandes" (WT/2LFFP 15/09). The authors thank the DFKI GmbH, and Saarland University for additional funding and for providing the necessary infrastructure, and E. Arzt for his support through INM

a)

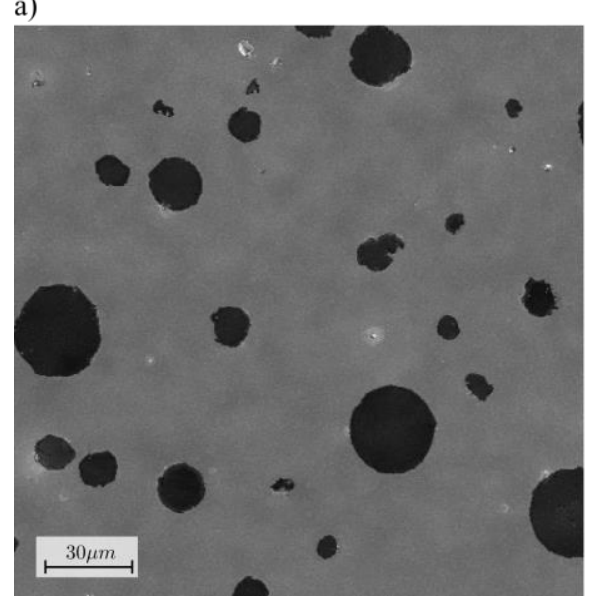

b)

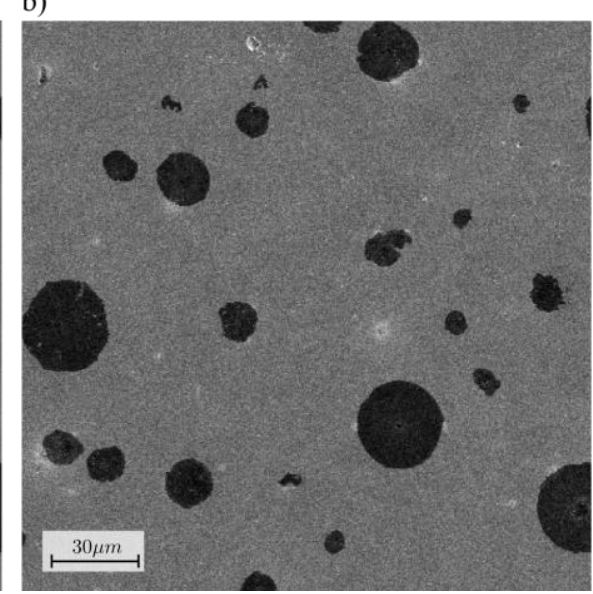

c)

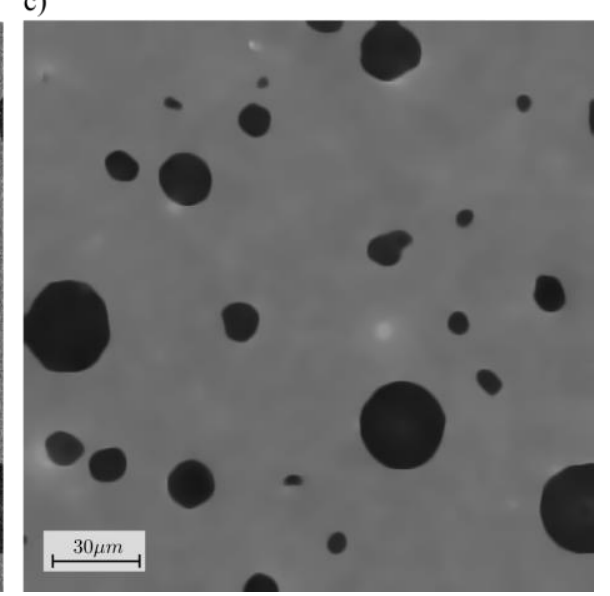

Figure 1. Overall performance of the adaptive sampling method. a) Image recorded with our method using an average pixel-dwell time of $3 \mu$ s (initial scan $300 \mathrm{~ns}$, sparse scan $27 \mu \mathrm{s}$ for $10 \%$ of pixels). b) Image recorded with uniform sampling at the same pixel-dwell time ( $3 \mu$ s per pixel). c) Image recorded at $3 \mu$ s per pixel and filtered using an anisotropic diffusion operator. 